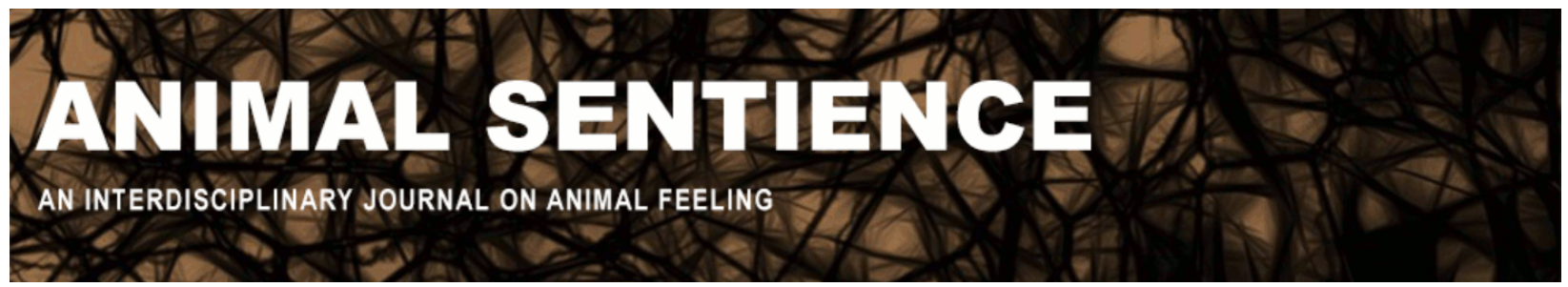

Brown, Culum (2017) A risk assessment and phylogenetic approach. Animal Sentience 16(3)

DOI: $10.51291 / 2377-7478.1219$

Date of submission: 2017-10-11

Date of acceptance: 2017-10-16 (c) 


\title{
A risk assessment and phylogenetic approach
}

\author{
Commentary on Birch on Precautionary Principle
}

\author{
Culum Brown \\ Department of Biological Sciences \\ Macquarie University
}

\begin{abstract}
The precautionary principal is often invoked when talking about the evidence of sentience in animals, largely because we can never be certain what any animal is thinking or feeling. Birch (2017) offers a preliminary framework for the use of the precautionary principal for animal sentience combining an epistemic rule with a decision rule. I extend this framework by adding an evolutionary phylogentic approach which spreads the burden of proof across broad taxonomic groups and a risk assessment component which magnifies the likely impact by the number of animals involved.
\end{abstract}

Culum Brown studies behavioural ecology of fishes with a special interest in cognition, personality and laterality. He is Associate Professor of vertebrate evolution at Macquarie University, Co-Editor of the volume Fish Cognition and Behavior, and Editor of the Journal of Fish Biology. https://thefishlab.com

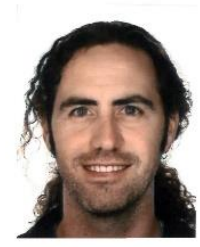

In the target article, Jonathan Birch (2017) outlines a practical framework for the use of the precautionary principal when it comes to considering the evidence that an animal is sentient and the decision to take action when formulating animal protection legislation. The Animal Sentience Precautionary Principle (ASPP) states that "Where there are threats of serious, negative animal welfare outcomes, lack of full scientific certainty as to the sentience of the animals in question shall not be used as the reason for postponing cost-effective measures to prevent those outcomes." His suggested approach is to combine an epistemic rule (BAR) with a corresponding decision rule (ACT) and provides a practical way to implement ASPP in the context of animal welfare. The overall framework is reminiscent of risk assessment. The approach is commendable because it begins to provide a practical framework that practitioners and researchers can use and refine.

In the past, I have called upon the precautionary principal as it applies to the inclusion of fish in animal protection legislation (Brown 2016). In doing so, I was not reaching out to the converted but rather to those who still harbour doubts. I have pointed out, as have many others (Wadiwel 2016), that there are no absolute truths in the field of animal sentience because we can never be $100 \%$ certain what another animal is feeling or thinking. This applies equally to humans (Avramides 2001). As far as I'm concerned, the multiple, independent lines of multidisciplinary evidence supporting pain in bony fish is more than sufficient to include them in our moral circle (Brown 2015; Sneddon et al. 2015). 
Birch makes an interesting suggestion that we should aim to examine the evidence for sentience for at least one representative for each order to achieve broad taxonomic coverage. $\mathrm{He}$ also sets a relatively low BAR - just one credible indicator of sentience for any given species. Personally, I would prefer a higher BAR, perhaps 2 or more independent lines of evidence to ensure there is little doubt. Assuming we can agree on the appropriate taxonomic level, Birch provides a decent framework with which one can begin to accumulate evidence. Within teleost fishes, for example, we are trying to examine more than half the vertebrate diversity on the planet, which is divided into 37 orders (Betancur-R et al. 2017; www.deepfin.org). Far too few have been examined to date largely because scientists tend to focus on model organisms (e.g., salmononiformes). But by Birch's definition, there is no doubt that those that have been studied to date would proceed to stage 2 - ACT - and thus be granted protection (Sneddon 2015).

Table 1. A theoretical risk assessment framework for determining the likely outcome of mistreating fish in the absence of mitigating actions. Likelihood refers to the scientific evidence that fish are sentient, feel pain and suffer accordingly. Consequences are the number of individual animals involved.

\begin{tabular}{|c|c|c|c|c|c|c|}
\hline & \multicolumn{5}{|c|}{ Consequences } \\
\hline & & $100 s(1)$ & $1000 s(2)$ & Millions (3) & Billions (4) & Trillions (5) \\
\hline \multirow{5}{*}{ 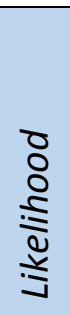 } & $\begin{array}{l}\text { Almost } \\
\text { Certain (5) }\end{array}$ & & & & & Catastrophic \\
\hline & Likely (4) & & & High & & \\
\hline & Possible (3) & & Moderate & & & \\
\hline & Unlikely (2) & & & & & \\
\hline & Rare (1) & Low & & & & \\
\hline
\end{tabular}

As noted, the framework that Birch presents is reminiscent of risk assessment procedures but he is missing the Consequences component. For those who are familiar with risk assessment, one usually generates a risk matrix based on a combination of the likelihood of a risk occurring and the consequences (Table 1). In this, perhaps more familiar framework, we can set a scene for animal welfare legislation which has similarities to Birch's approach. Here the Likelihood of risk could be determined by various levels of scientific evidence (BAR), and the consequences could be determined by the number of animals involved. In the case of fish, the evidence is substantial (Likely or Almost Certain) and the number of animals involved is in the trillions. Thus, the associated risk category is Catastophic (Table 1). In other words, in the absence of any mitigating actions (i.e., appropriate animal welfare legislation to protect fish from harm), our current treatment of fishes has catastrophic consequences for animal welfare. One can easily change the scale of this approach to the order level; in that case, the corresponding risk category will vary considerably, since some fish orders are more heavily exploited than others. 


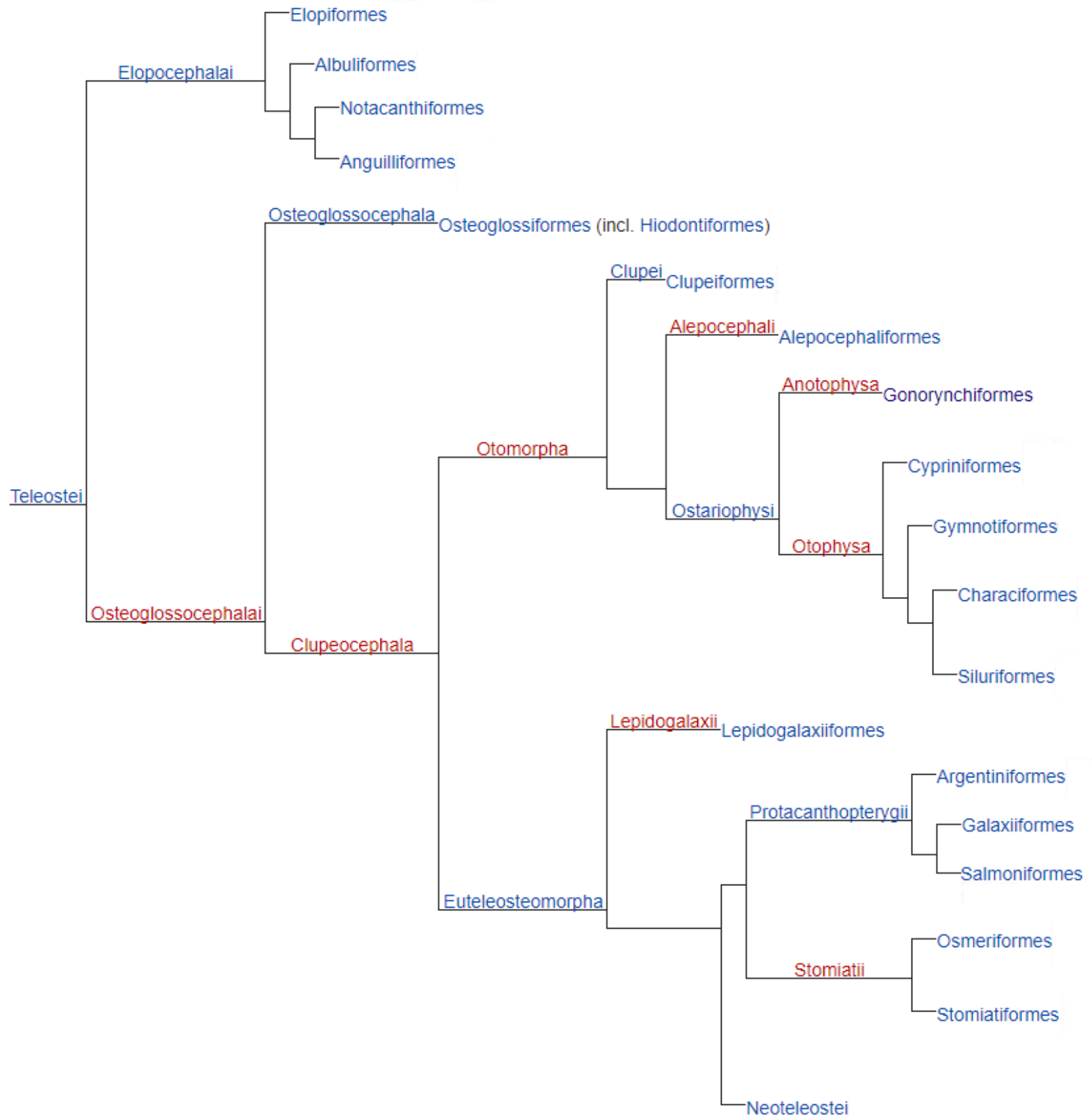

Figure 1. Phylogeny of extant teleost orders (based on www.deepfin.org).

I would add to Birch's thesis that we can also use the precautionary principal in viewing the problem through the eyes of an evolutionary biologist. Evolution tends to be highly conservative when it comes to traits under heavy selective pressure. Sentience and the ability to feel pain are good examples given the inherent fitness benefits. Closely related taxa tend to share traits through common decent (shared derived characters). Thus, if we know a trait exists in just a few orders within a phylogeny, we can use phylogenetic inference to determine its likely distribution 
in the phylogeny as a whole. Take the following example (Figure 1): If we had evidence that members of the Salmoniformes (e.g., Atlantic salmon) and the Cypriniformes (e.g., zebrafish) were sentient, then in the absence of any other information, we would conclude that all members of the Clupeocephala are sentient - that is, the trait is ancestral. The alternative, that the trait evolved twice independently, is less parsimonious. Thus, we do not necessarily need complete coverage of clades to be reasonably certain of the distribution of a trait as Birch suggests. Obviously, the more data we have, the greater the certainty. In the case of teleost fish, placing our existing knowledge in a phylogenetic framework would lead us to conclude that sentience is widespread not only in fishes but in all vertebrates.

Another nice example is the distribution of nociceptors in animals, which can be traced back at least as far as the Annelids. Comparative studies using a wide variety of model organisms have shown similarities in nociception from invertebrates through to humans (Smith \& Lewin 2009). To date, however, studies have failed to identify C-fibres in sharks and rays (Coggeshall et al. 1978; Leonard 1985; Snow 1993). This lack of evidence has been used as evidence of lack - a fundamental misinterpretation of scientific method - and has been taken to suggest that sharks and rays cannot feel pain. But the fact that nociception occurs in jawless fish (agnathans; Mathews \& Wickelgren 1978; Pastor et al. 1996) as well as in bony fish (teleosts; Sneddon 2015) - not to mention the rest of the vertebrates - suggests the most parsimonious explanation is that sharks do have these capacities but that we have yet to understand that the receptors or the fibers we have identified operate in a novel manner. The alternative explanation is that elasmobranchs have lost the ability of nociception, and one would have to come up with a very convincing argument for the adaptive value of such a loss in a single taxon in the entire animal kingdom.

\section{References}

Avramides, A. (2001) Other Minds, London: Routledge.

Betancur-R, R., Wiley, E.O., Arratia, G., Acero, A., Bailly, N., Miya, M. Lecointre, G. \& Orti G. (2017) Phylogenetic classification of bony fishes. BMC Evolutionary Biology 17, 162.

Birch, J. (2017) Animal sentience and the precautionary principle. Animal Sentience 16(1).

Brown, C. (2015) Fish intelligence, sentience and ethics. Animal Cognition 18, 1-17.

Brown, C. (2016) Fish pain: An inconvenient truth. Animal Sentience 3(32).

Coggeshall, R.E., Leonard, R.B., Applebaum, M.L. \& Willis W.D. (1978) Organization of peripheral nerves of the Atlantic stingray, Dasyatis Sabina. Journal of Neurophysiology 41, 97-107. 
Leonard, R.B. (1985) Primary afferent receptive field properties and neurotransmitter candidates in a vertebrate lacking unmyelinated fibres. Progress in Clinical Research 176, 135-145.

Matthews, G. \& Wickelgren W.O. (1978) Trigeminal sensory neurons of the sea lamprey. Journal of Comparative Physiology. A, Neuroethology, Sensory, Neural, and Behavioral Physiology 123, 329-333.

Smith, E.S.J. \& Lewin, G.R. (2009) Nociceptor: A Phylogenetic view. Journal of Comparative Physiology. A, Neuroethology, Sensory, Neural, and Behavioral Physiology 195, 1089-1106.

Sneddon, L.U. (2015) Pain in aquatic animals. Journal of Experimental Biology 218, 967-976.

Snow, P.J., Renshaw, G.M.C. \& Hamlin, K.E. (1993) Localization of enkephalin immunoreactivity in the spinal cord of the long-tailed ray Himantura fai. Journal of Comparative Physiology 367, 264-273.

Wadiwel, D. (2016) Fish and pain: The politics of doubt. Animal Sentience 3(31). 